DOI: 10.21767/2171-6625.1000191

\section{Blindsight: The Anatomical and Functional Post-Injury Neural Plasticity of The Visual System}

\section{Abstract}

The "blindsight" is the demonstrated ability of some patients with cortical blindness to respond to a visual stimulus presented in the corresponding visual field area, without perceiving it consciously. Our study was based on the use of neural tracers injected into the lateral geniculate body of animals from which the primary visual cortex was removed at birth. The neurons of the lateral geniculate body that survived the deprivation of their target projected elsewhere and probably underwent a reorganization of their efferent. Inserting the tracer in the neurons of the lateral geniculate body, our intention was to analyze the reorganization and the distribution of axonal projections between the cortex and the thalamus. The second purpose of the study was to analyze the role of JNK (c-Jun N-terminal kinase) in the reorganization of neuronal fibers after cortical ablation. By administering the inhibitor peptide, D-JNKI-1, the purpose was to examine how the cortical afferents that came from the surviving cells in the lateral geniculate body after cortical ablation arranged themselves. The intent of these studies was to try to use to our advantage the possibilities that result from plastic potentials that the nervous system has, to a greater or lesser extent, during all of its life. By the use of inhibitors, such as D-JNKI-1 we have shown that as a result of a lesion the neuronal death can be reduced and that a greater number of cells can be kept alive, each of which is potentially capable of emitting projections able to regroup to compensate the losses caused by the injury.

Keywords: Blindsight; Neural plasticity; Visual system

\author{
Nuzzi R' ${ }^{1}$, Buschini E' ${ }^{1}$, \\ Monteu $F^{1}$ and Vercelli $A^{2}$ \\ 1 Eye Clinic Section, University of Turin, \\ Ophthalmic Hospital, Turin, Italy \\ 2 Department of Neurosciences, \\ University of Turin, Italy
}

\section{Corresponding author: Monteu F}

\section{francesca.monteu@gmail.com}

Clinica Oculistica Universitaria, Ospedale Oftalmico, via Juvarra 19, 10122, Torino, Italy.

Tel: +39 3479949826

Citation: Nuzzi R, Buschini E, Monteu F, et al. Blindsight: The Anatomical and Functional Post-Injury Neural Plasticity of The Visual System. J Neurol Neurosci. 2017, 8:3.

Received: April 20, 2017; Accepted: May 15, 2017; Published: May 17, 2017

\section{Introduction}

The "blindsight" is the demonstrated ability of some patients with cortical blindness to respond to a visual stimulus presented in the corresponding visual field area, without perceiving it consciously.

The studies on it began in 1936 with Kluver [1], followed by Keating [2], Mohler [3] and Schilder [4] in the 70s. As knowledge advances, it has been shown that blindsight is more common than thought. Indeed, it is commonly thought that people with blindsight are rarely seen, since the majority of patients with damage to the primary visual cortex, shows apparent total blindness in the corresponding area of the visual field. In fact it is necessary to specify that the blindsight can only be discovered using methods of investigation that are based on forced-choice questions, on neuroendocrine analysis, reflected or implied. Many patients with cortical blindness, if investigated with appropriate methods and visual stimuli appropriate to the spatial and temporal characteristics of blindsight, show some residual visual activities.

It has been shown by several studies that in case of damage to the primary visual cortex, with appropriate methods, it is possible a certain degree of functional recovery.

One of the most common approaches is to try to reduce the areas of scotoma by adopting specific exercises to stimulate the border area between vision and blindness [5]. It has been shown that it is possible to recover a conscious, visual function with the exercise of unconscious, implicit skills, typical of blindsight [6]. It is not inconceivable that the blindsight simply represents an operating system even in normal individuals, working in parallel to the conscious ways and from them, under normal conditions and in the absence of injury, overwhelmed [7]. Seen in this light the phenomenon would represent one of the clearest examples 
of dissociation between cognitive processes, conscious and unconscious [8].

Our study is based on the use of neural tracers injected into the lateral geniculate body of animals from which the primary visual cortex was removed at birth. The neurons of the lateral geniculate body that survived the deprivation of their target project elsewhere and probably undergo a reorganization of their efferent. Inserting the tracer in the neurons of the lateral geniculate body, our intention is to analyze the reorganization and the distribution of axonal projections between the cortex and the thalamus.

The second purpose of the study is to analyze the role of JNK (c-Jun N-terminal kinase) in the reorganization of neuronal fibers after cortical ablation. By administering the inhibitor peptide, D-JNK-1, the purpose is to examine how the cortical afferents that come from the surviving cells in the lateral geniculate body after cortical ablation arrange themselves.

JNK could be an important therapeutic target in case of perinatal central nervous system lesion, because it would be able to decrease the neuronal death due to the damage, favoring the processes of neuronal connections reconstruction using pathways different from normal. Therefore, visual function might be preserved during extended cortical lesions, enhancing what physiologically occurs in cases of blind sight.

On the other hand it is also proposed to detect possible side effects of a treatment whose aims is to block JNK. JNK in fact, is not only important in apoptotic processes, but it is also essential in physiological and regenerative mechanisms, which include formation and growth of axons, brain development, dendritic architecture and regeneration of nerve fibers in response to damage.

\section{Methods}

For the realization of our study we employed six Wistar albino rats, bred in the enclosure of the Department of Anatomy, Pharmacology and Forensic Medicine of the University of Turin. The animals were reared in cages of an appropriate size, maintained under controlled temperature conditions $\left(22-24^{\circ} \mathrm{C}\right)$ and with light dark cycles of 12 hours. Unhindered and continuous access to water and food was guaranteed. All experiments were conducted in accordance with the guidelines drawn up by the Italian Ministry of Health (DL 116/92) and in full respect of the animals, trying to minimize their suffering.

The animals used were as follows:

- Two rats were subjected only to the removal of the visual cortex;

- The ablation of the visual cortex and the inoculation of D-JNKI-1 peptide were performed in two rats;

- Two rats of 7 days (P7) were sacrificed as controls.

The day after the birth (P1), the rats were anesthetized by hypothermia with ice for 5 minutes. A longitudinal incision along the scalp was performed in a median position, to expose the cranial theca. A hole in the skull in correspondence to the right visual cortex was performed with a needle and the underlying cortical material was aspirated with a glass Pasteur-type pipette. The scalp was then sutured by a $4 / 0$ sterile silk thread and the rats were placed on a heating pad for a few minutes. After full recovery of consciousness and vitality, they were put back with their mother.

Rates of $10 \mu \mathrm{l}$ of D-JNKI-1 1mM (XG-102, "Xigen Lausanne CH"), corresponding to $0.5 \mathrm{mg}$ of peptide, stored at $-80^{\circ} \mathrm{C}$, were used. At the time of use, the aliquots were further diluted with distilled water as necessary to administer an amount of $0.3 \mathrm{mg} / \mathrm{kg}$ of D-JNKI-1. The injections were practiced intraperitoneally, after mild hypothermia, in the $\mathrm{P} 1$ chosen rats, using $0.5 \mathrm{ml}$ syringes. After a wait of 30 minutes the ablation of the visual cortex was performed.

On the seventh day of life (P7), the rats were sacrificed with an overdose injection of anesthetic, intraperitoneally ( $3 \mathrm{~g}$ of chloral hydrate in $10 \mathrm{ml}$ of physiological solution). After immobilization on the metal support, a longitudinal cervico abdominal incision was performed and the sternum was removed. After heart exposure, a small cut in the right atrium was practiced to create an open circulation, and the needle of a butterfly attached to a syringe filled with perfusion buffer was inserted in the left ventricle. After a slow wash with $5-10 \mathrm{ml}$ of perfusion buffer the latter was replaced with $4 \% \operatorname{PAF}$ (4\% paraformaldehyde), in a dose almost equal to the rats weight. After the rats were scalped, bone marrow was sectioned to the occipital base and the skull was opened longitudinally along the median fissure to allow the removal of the brain. The excised sample was immersed in $4 \%$ PAF and stored at $4{ }^{\circ} \mathrm{C}$ for at least 2-3 hours.

We used powder Dil (1',1-dioctadecyl-3,3,3',3'tetramethylindocarbocyanin-perchlorate, "Molecular Probes INC., Or USA"), a lipophilic tracer which was seen as a red florescent when examined with rhodamine filter. After the extraction the brain was frontally sectioned at the level of the anterior poles to create a support base, while at the caudal level was sectioned posteriorly to the superior collicoli until the lateral geniculate bodies were exposed; inside the lateral geniculate bodies some Dil crystals were introduced using a fine needle. The samples obtained were therefore immersed in $4 \% \mathrm{PAF}$ and placed in a heater at a constant temperature of $37^{\circ} \mathrm{C}$ for a variable time of $15-22$ days (until the cortical neurons were marked). Thereafter the samples were stored in a $4 \% \mathrm{PAF}$ solution at a temperature of $-4^{\circ} \mathrm{C}$ until their usage.

Two Wistar rats, born and raised without experimental manipulation, were sacrificed on the seventh day and their brains were prepared and stored using the same procedure.

After a 24 hours washing with saline perfusion buffer (PBS 1x), the samples were included in a media prepared with $70 \mathrm{ml}$ of distilled water, $15 \mathrm{ml}$ of $99 \%$ glycerol and $15 \mathrm{~g}$ of gelatin (an heterogeneous mixture of water soluble proteins of bovine skin, derived from lime-cured tissues) and cut using Leica VT $1000 \mathrm{~S}$ vibratome to obtain sections of $100 \mu \mathrm{m}$ of thickness, gathered in PB $0.1 \mathrm{M}$ windows. The sections were counterstained with bisbenzimide, 
a DNA intercalant that permits to distinguish the six layers of the brain cortex. After a 20 min incubation with reactant multiple washes with PB $0.1 \mathrm{M}$ were performed to completely remove the dye. The sections were therefore prepared on microscope slides in a $0.1 \mathrm{M}$ PB and $99 \%$ glicerole solution.

The slides obtained were examined using a fluorescence microscope "Nikon eclipse E8000" with rodamine filter, to study the red florescence of Dil, and with UV filter to study the blue florescence of bisbenzimide.

The photographs were taken with a digital camera, "Nikon Coolpix E995", with different magnifications (2x, 4x and 10x).

The sections were examined with "Bioscience's Neurolucida version 7" software MBF program integrated with a fluorescence microscope, "Nikon Eclipse E700", usually used to map histological sections and track nerve fibers. During our study we analyzed in detail three sections for each animal.

With a $4 x$ magnification the outline of the sections was drafted and using different "user lines", areas corresponding to the cerebral cortex of both hemispheres were demarcated. More "user lines" were used to divide the cortical upper granular layers (I-IV) from the under granular ones (V-VI). Within these spaces, with 10x magnification, the cell bodies visible in fluorescence were marked using different "markers" in the two hemispheres.

The quantitative information was examined using "Neurolucida Explorer", the 3D viewer and morphometric analysis software. The data were related to:

1. The total number of "marker" for "user line";

2. The length of each "user line" and the corresponding areas enclosed inside them.

\section{Results}

The primary visual cortex ablation is linked to the lateral geniculate bodies neurons degeneration, due to the axotomy of the fibers projecting towards the cortex. Some studies demonstrated that it was the neuronal death as the cause of the degeneration [9]. The purpose of previous experiments performed in our laboratory using Wistar rats was the quantification of the phenomenon by calculating the number of apoptotic cells that were found in the lateral geniculate bodies 24 hours after the ablation of the visual cortex in the first day after the birth. Other rats, however, were sacrificed 7 days after the ablation and the count of surviving cells was subsequently effected. In some of these animals the D-JNKI-1 peptide was administered before cortical ablation, while in others rats only the incision along the scalp was performed, without cortex damage. Histological sections obtained by microtome were stained with "Nissl" (basic aniline) and examined by a microscope interfaced with a computer using the "Stereo-Investigator" program.

In the sections photographs colored with "Nissl" of the samples sacrificed after 24 hours could be observed in the ipsilateral to the ablation lateral geniculate body numerous apoptotic bodies, which were not present in the cases with D-JNKI-1 and almost absent in controls. The results of the stereological count in fact showed that in rats with ablation of the visual cortex the count of apoptotic cells in the lateral geniculate body of the same side of the lesion after 24 hours was very high, compared to the controls, while the animals treated with D-JNKI-1 showed an intermediate value between the rats with the only ablation and the controls. Both opposite side of the lesion hemispheres cells number were comparable to those of controls, but they were particularly low in the treated animals.

The photographs staining of the samples sacrificed after 7 days showed a higher cellularity in the treated animals compared to the one observed in rats subjected only to the ablation.

The neuronal bodies count showed that the neuronal survival in the hemisphere on the side of the lesion in the samples subjected to the cortical lesion was less than the one in the contra lateral hemisphere and was also less than the one of the controls. The counts in animals treated with D-JNKI-1 peptide were higher, in both hemispheres.

In our study, the lipophilic tracer Dil was used as a retrograde marker in samples fixed with $4 \%$ PAF to analyze the pathways between the cerebral cortex and the thalamus. The tracer, inserted into the lateral geniculate body, by diffusion through the axon terminals of the cortico-thalamic projections showed the bodies and the neuronal dendrites in the cortex. Studying their quantification, characterization and localization we tried to understand if and how there has been a reorganization of the lateral geniculate bodies neurons projections, after visual cortex ablation and therefore depriving the neurons of their natural target.

The six animals used in the study were divided into three groups of two (controls, visual cortex ablation, ablation + D-JNKI-1). Three brain coronal sections were analyzed for each sample and the cortical areas of interest and the number of labeled cells were calculated, obtaining the cells density data. For each animal we calculated the averaged results of the three sections and finally a unique average was calculated for each group, in order to obtain comparable data.

\section{Controls}

Distribution of the neuronal bodies in the cortex: In the photographs taken by the camera attached to a fluorescence microscope many cell bodies labeled with Dil were seen, (Figure 1c) with a mostly under granular layer pattern of distribution and almost totally located in layer V. In particular thalamus cortical neurons were located for the most part in the deepest part of the fifth layer and a few in the most superficial part of the sixth layer. Their distribution was uniform in the fifth layer, while in the sixth, the labeled cells were distributed in groups. (Figure 2c) These observations were confirmed by the reconstructions obtained with the software "Neurolucida" (Figure 1c) and the data analysis showed that the under granular layer density values (293.52 cells $/ \mathrm{mm}^{3} \pm 11.92$ ) were almost three times than the upper granular layer values (77.61 cells $/ \mathrm{mm}^{3} \pm 26.50$ ) (Figure 3). 

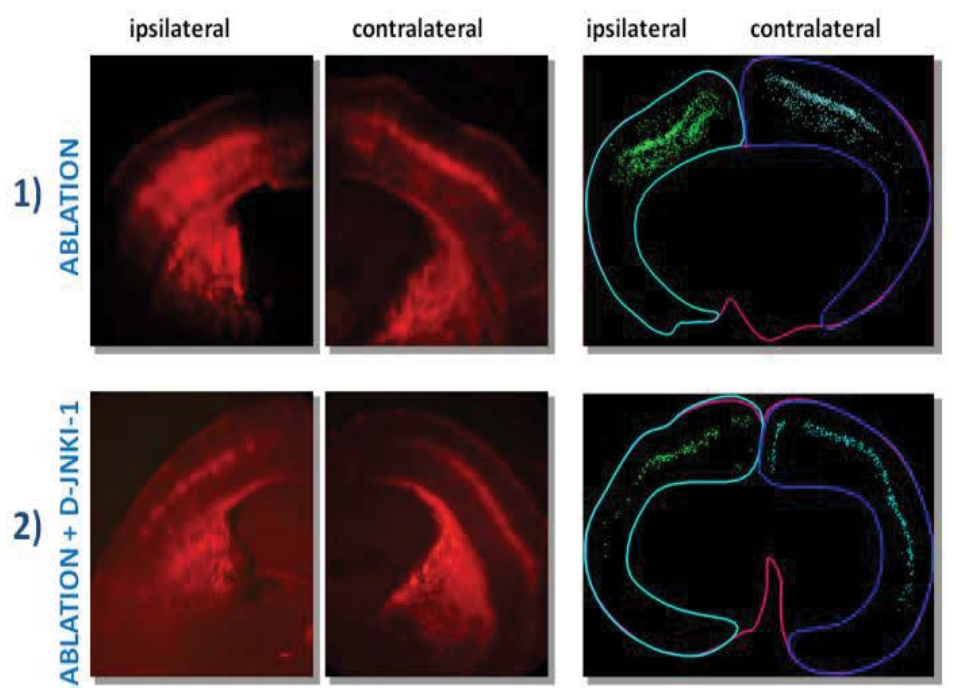

3)
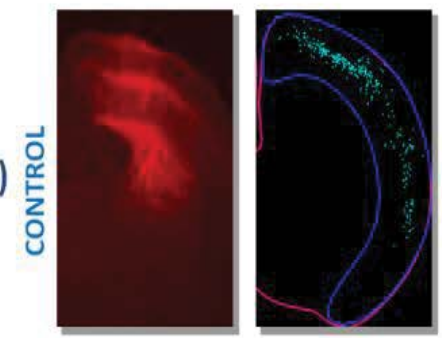

On the left: Fluorescence microscopy Dil marked pictures of neuronal bodies of cortico thalamic projections on the left side.

a. Visual cortex ablation: in the ipsilateral to the lesion hemisphere there is a greater cell density and disorganization than in the contralateral hemisphere.

b. Ablation + D-1-JNKI administration: both the hemispheres have the same characteristics. They have a more homogeneous distribution and there is a greater cell density in the contralateral to the lesion hemisphere.

c. Control: the distribution appears homogeneous and uniform.

On the right side: Neurolucida images on the right side. With Neurolucida images, the cortical localization of the neuronal bodies is marked.

a. Visual cortex ablation: in the ipsilateral to the lesion hemisphere the markers have a disposition similar to the contralateral one, but they are a higher number.

b. Ablation + D-1-JNKI administration: the distribution of the markers in ipsilateral and contralateral hemispheres is similar, but the markers are in higher number in the contralateral one.

c. Control: the marker has a distribution similar to the controlateral to the lesion hemisphere of the rat subjected only to the ablation.

Figure 1 Cortico-thalamic projections of the neuronal bodies marked with fluorescence and Neurolucida images.

As regards tangential direction distribution, the labeled neurons were localized mainly in the somatosensory cortex and in lesser number in the primary motor cortex.

Neuronal density in cortical areas of interest: The total average density of the labeled cells in the 6 hemispheres in 6 different sections of the two control rats amounted to 205.38 cells $/ \mathrm{mm}^{3}$ \pm 7.28 .

Marked neuronal type: The nerve cells marked by the tracer and examined at high magnification microscope corresponded to a pyramidal phenotype, the neuron typology normally present in these cortical areas (Figure 4c). The thalamus-cortical pyramidal neurons in layer $\mathrm{V}$ were characterized by a prominent apical dendrite that reached the layer I up to the pia mater, where it branched into a terminal tuft with an extension in the tangential direction. The apical dendrite had also various oblique branches with an almost horizontal orientation. The basal dendrites were 3-5 for each cell and radiated out horizontally to form a dendritic plexus clearly visible at low magnification, which extended throughout the fifth layer and in the most superficial part of the sixth layer. The dendrites had numerous spines with a immature morphology (threadlike or short and herring bone). Axons, much thinner then dendrites, were directed perpendicular to the surface to the white matter and they branched out into horizontal axons in the sixth layer; Then, in the white matter they headed laterally towards the thalamus.

\section{Visual cortex ablation}

\section{Distribution of neuronal bodies in cortex:}

- Ipsilateral to the lesion hemisphere: The marking was almost totally under granular $\left(867.41\right.$ cells $/ \mathrm{mm}^{3} \pm 175.91$ vs 25.74 cells $/ \mathrm{mm}^{3} \pm 3.93$ ), mainly located in layer $\mathrm{V}$ and partially in layer VI (Figure 2a). The cells were well bonded to each other and located mainly at the level of the somatosensory cortex, while only a few neurons could be demonstrated in the motor cortex.

- Contralateral to the lesion hemisphere: The labeled cells were mainly distributed in under granular layers (338.71 cells $/ \mathrm{mm}^{3} \pm 147.90$ vs 12.86 cells $/ \mathrm{mm}^{3} \pm 7.57$ ) as in the controls, but they were more spread and divided between the fifth and sixth layer. The greater number of neurons was in the somatosensory cortex, but also in this case some neurons were marked at the level of the motor 

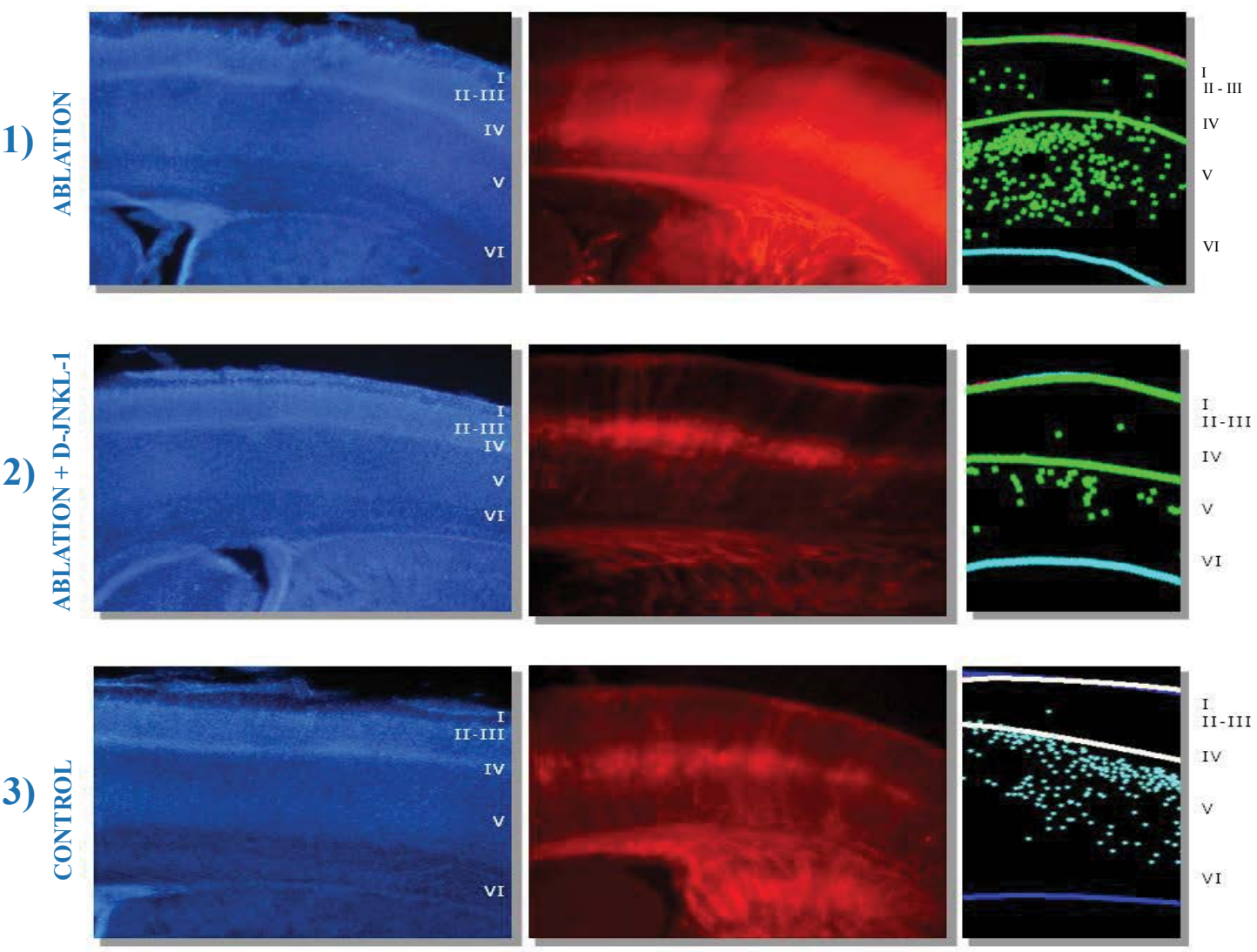

Direct comparison between fluorescence microscopy pictures of sections marked with bisbenzimide filtre on the left side, with Dil in the centre and Neurolucida images on the right side. The distribution is predominantly a under granular one (V, VI layers) in all of the three groups.

a. Ipsilateral to the lesion hemisphere of a rat subjected only to ablation: the intense marking is predominantly in $\mathrm{V}$ and VI layers.

b. Ipsilateral to the lesion hemisphere of a rat subjected to ablation and D-1-JNKI administration: there are fewer marked neuronal bodies, predominantly distributed in the V layer.

c. Control section: the marking distribution is similar to the one of the rat subjected to ablation + D-1-JNKI administration, but with a larger number of cells.

Figure 2 Upper/under granular layer distributions (bisbenzimide/Dil/Neurolucida).

cortex.

\section{Neuronal density in cortical areas of interest:}

- Ipsilateral to the lesion hemisphere: The total average density of labeled cells present in 6 hemispheres of the 2 rats with ablation of the visual cortex amounted to 509.50 cells $/ \mathrm{mm}^{3} \pm 92.50$ (Figure 1a).

- Contralateral to the lesion hemisphere: The total average density was equal to less than the half of the ipsilateral value, of 193.50 cells $/ \mathrm{mm}^{3} \pm 69.50$. Marked neuronal type: Even in animals with ablation pyramidal cells were observed, in both hemispheres (Figure 4a).

\section{Ablation of the visual cortex + administration of D-1-JNKI}

\section{Distribution of neuronal bodies in cortex:}

1. Ipsilateral to the lesion hemisphere: The labeled cells had a very clear distribution, (Figure 1b) they had a under granular localization ( 151.90 cells $/ \mathrm{mm}^{3} \pm 43.13$ vs. 12.73 cells $/ \mathrm{mm}^{3} \pm 2.27$ ), with cells located almost exclusively in the fifth layer. It could be noted at this level a very ordered and linear arrangement (Figure $\mathbf{2 b}$ ). The marking was mainly in the somatosensory cortex, while neurons were less visible in the motor cortex.

2. Contralateral to the lesion hemisphere: Almost all cell bodies had a under granular pattern (195.98 cells $/ \mathrm{mm}^{3} \pm$ 97.55 vs. 4.95 cells $/ \mathrm{mm}^{3} \pm 2.04$ ), and in this case it was almost totally in the fifth layer. 


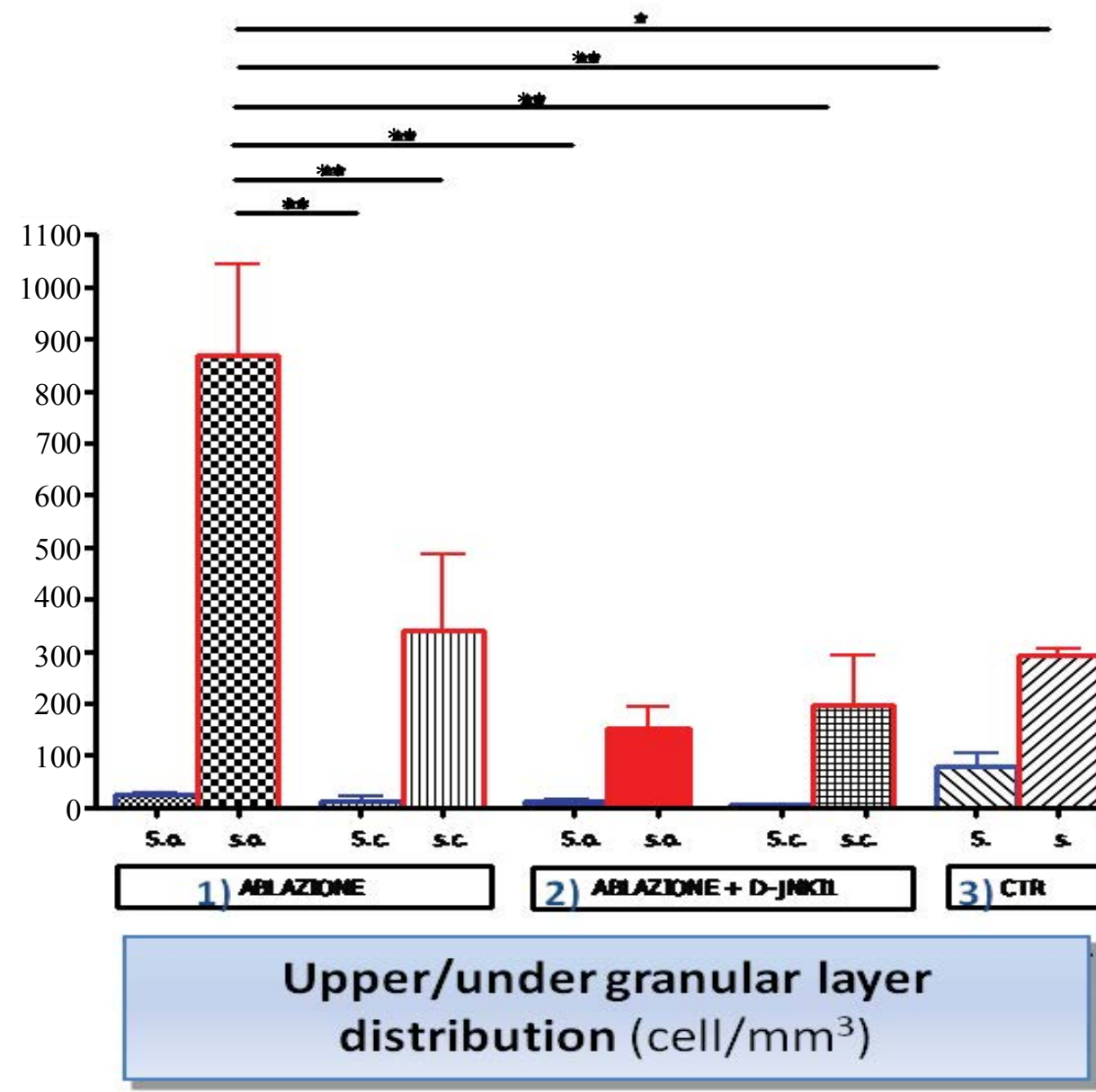

The mean values of cell density $\left(\mathrm{cell} / \mathrm{mm}^{3}\right)$ in the upper and under granular layers are indicated, respectively in rats subjected to 1 ) the only visual cortex ablation, 2) ablation + D-1-JNKI administration, 3) controls, studying ipsilateral and contralateral to the lesion hemispheres for each group.

(Legend: $\mathrm{S}=$ Upper Granular, $\mathrm{S}=$ Under Granular, $\mathrm{O}=$ Ipsilateral to the Lesion Hemisphere, $\mathrm{C}=$ Controlateral to the Lesion Hemisphere, $\mathrm{Ctr}=$ Controls, ${ }^{* *}=p \leq 0.001,{ }^{*}=p \leq 0.05$ ).

a. Visual cortex ablation: Ipsilateral hemispheres: the cell density is higher in the under granular layers. -contralateral hemispheres: the cell density is higher in the under granular layers, but the values are lower than in the ipsilateal hemispheres.

b. Ablation + D-1-JNKI administration: Ipsilateral hemispheres: the major density is in the under granular layers, but the values are lower than in the sections subjected only to the ablation. -contralateral hemispheres: the density is significantly higher in the under granular layers. The values are lower than the ones in rats subjected only to the ablation, but higher than the ones in the ipsilataral hemisphere.

c. Controls: The under granular density is higher than the upper granular one, but the upper granular density is higher than the same in the other groups.

Figure 3 Upper (I-IV)/under (V-VI) granular layer distribution (cells counting).

The distribution appeared very linear throughout the cortex, even if the cells showed greater dispersion when compared to those marked in the ipsilateral to the lesion hemisphere. They were mainly located in the somatosensory cortex, but they were also present in the motor cortex.

\section{Neuronal density in cortical areas of interest:}

1. Ipsilateral to the lesion hemisphere: The average density in the two animals was equal to 84.19 cells $/ \mathrm{mm}^{3} \pm 21.24$.
2. Contralateral to the lesion hemisphere: In contrast to what observed in the other groups of animals the average density value in the contralater to the lesion hemisphere was greater than that ipsilateral one, and was equal to 190.64 cells $/ \mathrm{mm}^{3} \pm 20.40$ (about twice).

Marked neuronal type: Even in this group of animals, the labeled cells were pyramidal one, in both hemispheres (Figure $\mathbf{4 b}$ ). 

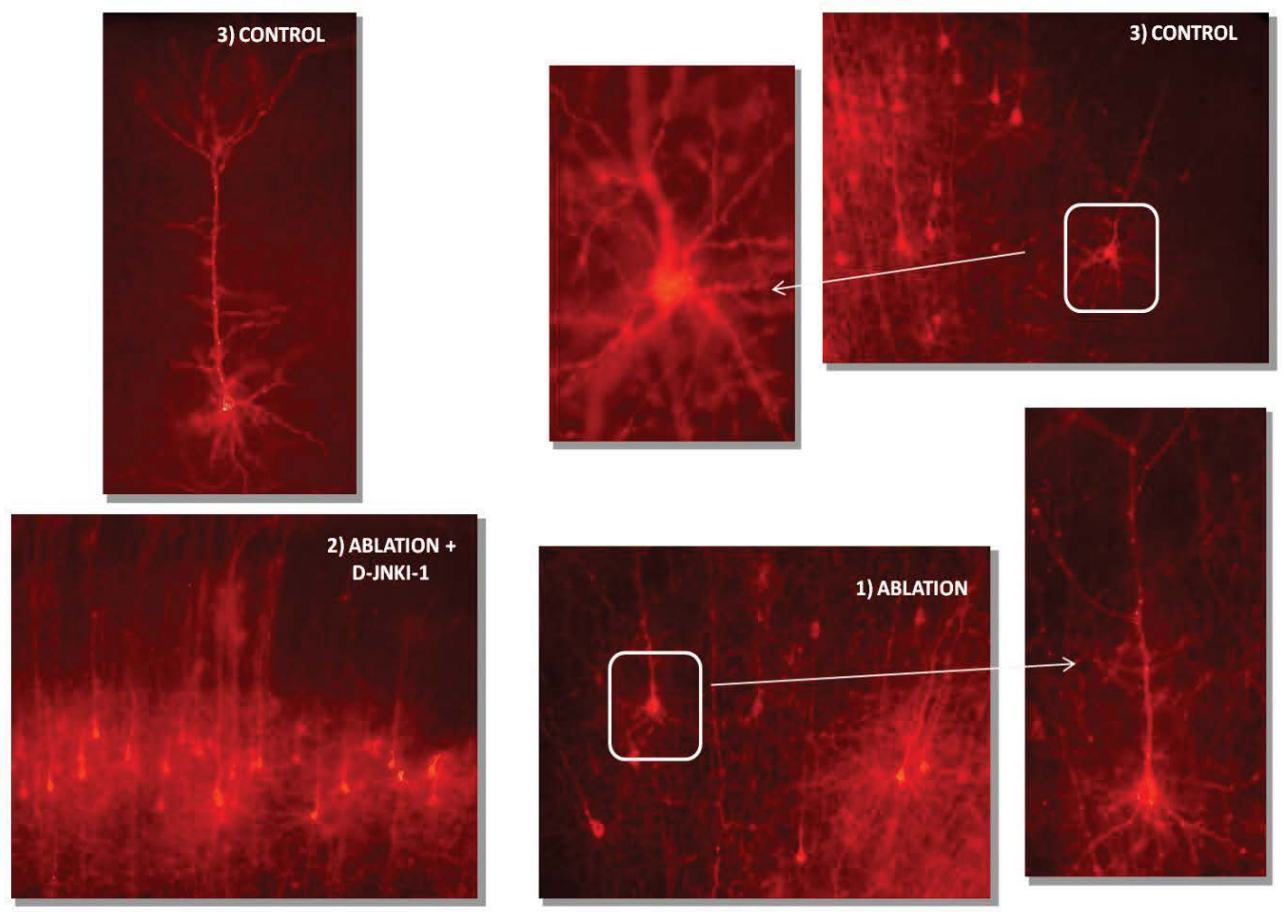

a. Pyramidal undergranular neurons in the $\mathrm{V}$ layer of the somatosensory cortex in the ipsilateral to the lesion hemisphere of a rat subjected only to the visual cortex ablation.

b. Undergranular neurons in the $\mathrm{V}$ layer of the somatosensory cortex in an ipsilateral to the lesion hemisphere of a rat subjected to ablation and D-1-JNKI administration.

c. Pyramidal neuron in the $\mathrm{V}$ layer of the motor cortex of a control rat.

Figure 4 Marked neuronal pyramidal type, in all of the three groups of the study. Dil marked cortical pyramidal neurons.

\section{Discussion}

The correct function of the visual system depends on the presence of adequate connections between retina and primary visuals brain centers. The final morphological organization depends on various kind of phenomena, both progressive and regressive, that take place during the development. During the initial stages the nerve cells starts to proliferate and to migrate, creating numerous contacts between them, even more than the ones needed during the adulthood. In the following stages the plastic effect depends principally on the neuronal regression, during which a part of the initial cells dies [10]. The surviving neurons selectively lose their dendrites, axons and exceeding synapses [11].

The physiological evolution procedure can be altered for pathological events or with experimental peripheral (eg. eyelid suture or eye enucleation) or central (primary visual cortex ablation) manipulations. The adult cerebral cortex is composed of six different layers, each one with a characteristic kind of neurons with its own distinct projection specificity [12]. The evolutive mechanism that creates this characteristic cortical morphology is not very understood, but evidences demonstrate that young neurons have more connections than the adulthood neurons [13]. Therefore during the development a cortical neuron have the potentiality to branches its collateral projections to a wide range of targets. In our study we showed that the early post natal plasticity causes cortex reorganization after cortical experimental ablation.

Particularly we discuss the following points: the thalamuscortical interconnection, the used methodology, the thalamuscortical neurons in the $\mathrm{V}$ layer, the link between plasticity and neuronal death, the link between neuronal death and survival, the thalamus-cortical projection reorganization and the D-JNKL-1 interpretation controversy.

Our study aimed to investigate the connection between thalamus and cortex, involved in the processing of sensory information. The periphery inputs have as the last neuronal terminal, before the cortex, the thalamus. The cortical neurons in V and VI layers which receive thalamic connections, send descending projections towards the same nuclei, creating an interconnection web. The cerebral cortex has the ability to dynamically influence the process modifying its own inputs [14].

After the visual cortex experimental ablation we caused a retrograde partial degeneration in the lateral geniculate body of the ipsilateral thalamus. We investigated the presence of projection reorganization of the surviving cells deprived of their natural target (Figure 5). Unfortunately Dil studies weren't able to distinguish the thalamus-cortical ascending projections because the Dil injection in the lateral geniculate body caused 

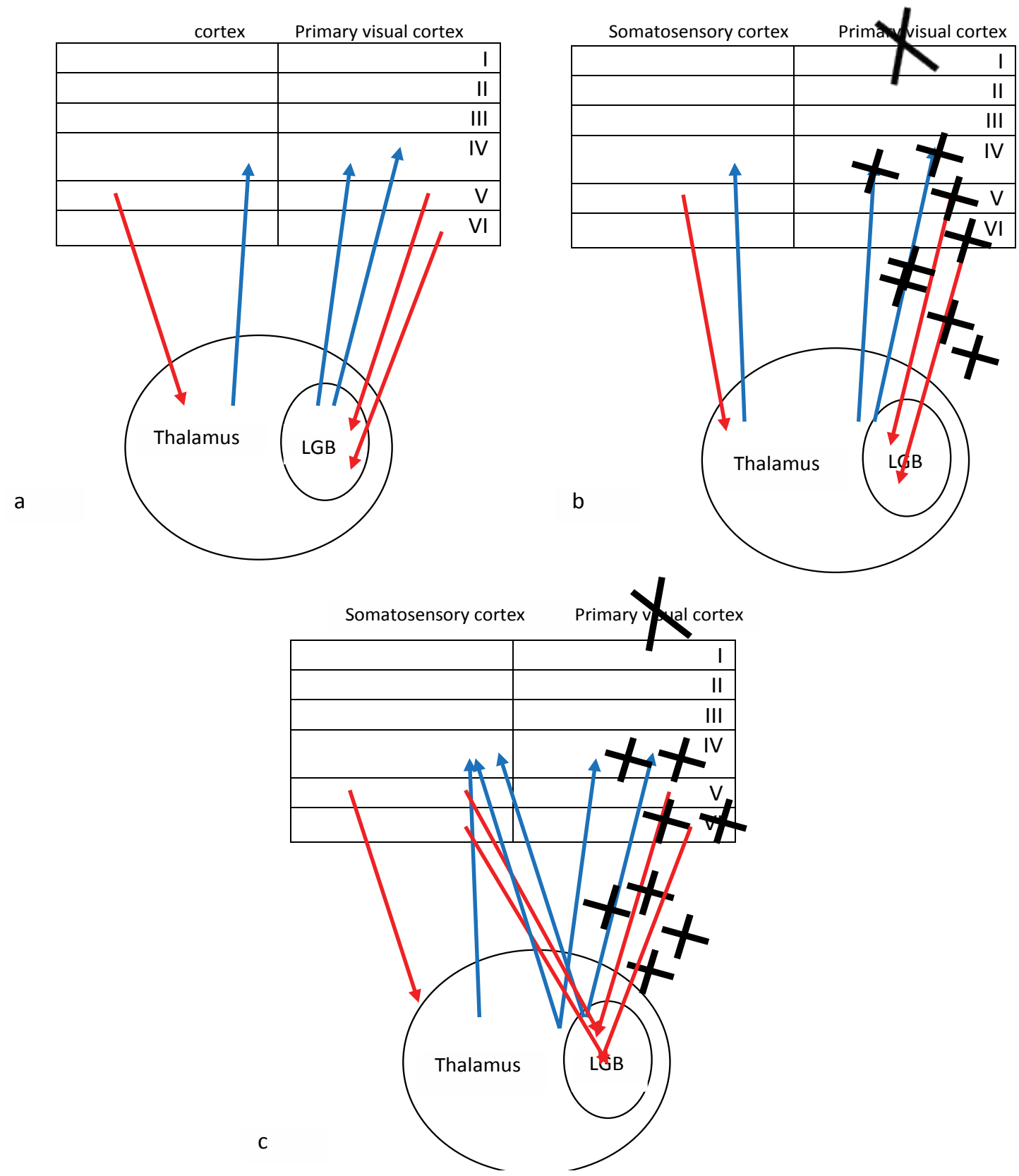

The red arrows represent the cortical-thalamic projections; the blue arrows represent the thalamic-cortical projections.

a. Controls.

b. Visual cortex ablation.

c. Re organization of the axon projections after visual cortex ablation + D-1-JNKI administration.

Figure 5 Diagram of the reciprocal connections between thalamus and cortex.

a retrograde diffusion along the axons terminals in the cortical thalamic projection marking neuronal bodies in the cortex.

However, the Dil tracer proved to be an efficient marker in vitro, allowing to insert precisely the crystals without a traumatizing surgical procedure. Since it is lipophilic, it spreads across phospholipid membranes and it marks the neuron. It has enabled us to visualize precisely the neuronal cell bodies, the axons, the dendrites, and also smaller details, as dendritic spines.
The Dil fixed tissues require particular procedure to obtain a clearer scan. The diffusion take place with a $2 \mathrm{~mm} /$ month velocity at ambient temperature, but it is possible to improve the velocity at higher temperatures. In our study the brains were stored in a stove at constant temperature for 4 weeks. The best method to cut is using the vibratome, because the paraffin inclusion procedure for the microtome interferes with the tracer and the freezing for the cryostat frequently damages the membranes 
(site where the marking is done). The sections were fixed in PBSglycerol to slow down the fluorescence decay [15].

The results were satisfactory, confirming similar studies reported $[16,17]$. The number of animals used was independent of the results obtained. In our laboratory, experimental trials are ongoing to overcome the problem of retrograde diffusion, as other Authors have studied [18], using in vivo injections of biotinylated dextran in the lateral geniculate body, an anterograde marker that should allow visualizing thalamus cortex projections in about 5 days.

Despite the long lasting immunohistochemical reaction needed to be visualized, the obtained marking is more stable because it doesn't emit fluorescence and it can be examined using a optical microscope at any time. Furthermore, it allows an optimal visualization quality of details, compare to lipophilic tracers [15].

In our study, using Dil, we focused on the morphology and on the cortical thalamic projection reorganization. The under granular layers of the cortex are composed of neurons with a pyramidal phenotype, with axons that emerge from the cortex. The cortical thalamic neurons belong to this type. The most represented cell population in our study is localized in V layer and it shows an aspect characteristic of cortical thalamic projections, as documented by many Authors $[19,20]$.

The Dil labeled neurons show a wide cell body and a typically pyramidal shape, with extended ramifications within the cortex. On the upper part of them there is a thick apical dendrite that along its course gives rise to collateral branches in the IV and $\mathrm{V}$ layer. Typically, the apical dendrite bifurcates itself in the III and IV layer and ascends up to layer I, where it branches into 2-4 primary branches, each of which ends in a large clump next to the pia mater. The axon gives rise to a fair number of long horizontal ramifications, which extends in the layers III and V, before it exits from the cortex to reach the thalamus.

As regard the neuronal plasticity, in the past we have studied the molecular pathways involved in neuronal death during the development of the visual system $[9,21]$ observing the key role of the activation of caspase 3 and caspase 8. More recently we have investigated the role of JNK as a molecule involved in neuronal death, upstream of the caspase 3 activation. The results so far obtained show that both the deafferentation that the deprivation of the target activate JNK in the superficial layers of the superior colliculus and of the lateral geniculate nucleus. The administration of an inhibitor of the JNK pathway, the D-JNKI-1 peptide, prevents the increase of the activation of caspase 3 during the cell death in both the primary visual centers.

Subsequently, on the contrary to what observed in the controls, in our models of cortical ablation it was observed in the lateral geniculate body high concentration of apoptotic bodies after 24 hours and a decrease of neuronal densities after 7 days. In the animals treated with D-JNKI-1 peptide the apoptotic bodies are present in lower number and the tardive cell density was higher. It is further confirmed from these studies that as a result of deafferentation, in neurons of the lateral geniculate body there is a massive cellular degeneration and that an effective protection against the neuronal death is obtained with the D-JNKI-1 peptide.

Through the studies mentioned above we deduced the JNK key role in neonatal neuronal death and that the inhibition of its activity protects the neurons that have lost their target. These neurons could than connect to other targets.

In our study after cortex ablation we observed many reorganization patterns of the cortical-thalamus efferences, that can be explained by multiple hypotheses. The D-JNK-1 role is non-univocal.

According to a theory postulated by Deschenes and colleagues in 1998 [22] the distribution of cortico thalamic projections originating from different cortical areas highly dependent on the extent of thalamic afferents. In the case of removal of the visual cortex it is plausible that the cells still alive inside the lateral geniculate body reorganize their efferent projections to other uninjured cortical areas.

The result is that the target areas of the cortex receive a higher number of connections than the normal ones, followed by a corresponding reorganization of the cortico-thalamic projections. In our ablated animal models we observed a cellular density increase principally of the somatosensory cortex of the experimentally manipulated hemispheres.

A plausible alternative hypothesis concern development mechanisms of the cerebral cortex. It is known that in the adults rats the neurons that projects to the thalamus originate from the layers V-VI. During the development the axons in layer $\mathrm{V}$ grow faster than the layer $\mathrm{VI}$ ones, reaching quicker the thalamus [16]. Therefore, in the newborn rats, the most important cortical-thalamus projections are in the layer $\mathrm{V}$. The final connections develop after the progressive grown of the projections from layer $\mathrm{VI}$ and the corresponding regression of the ones from layer $\mathrm{V}$. The cortical ablation experimentally obtained could be an obstacle to the elimination of the redundant connections of the layer $\mathrm{V}$ because the lateral geniculate body is an area that needs to be re-innervated. The development procedure could be altered and therefore the typical early postnatal disorganization could be maintained. In rats that have been injected with the D-JNKI-1 peptide the cortical efferences distribution seems more linear and organized than in the rats with only the cortical ablation. This particular morphological aspect could be explained by the fact that D-JNKI-1 besides being an inhibitor of the neuronal degeneration, is also a inhibitor of the remodeling due to the damn. For this reason we see a lower density of cortico thalamic projections and a linear arrangement. Seen in this light, the results observed in our experiments would seem a side effect of the peptide and may preclude a future therapeutic use.

To evaluate other possible applications concerning the observations considered it might be considered the duration of the effects of the peptide. It is assumed that D-JNKI-1 has a fairly long half-life (about 4 days), although unconfirmed. In this case it might be possible that sacrificing animals at a more mature age, the results of the reorganization happened after removal of the peptide could be seen. In this case, therefore, the more neuronal 
survival obtained thanks to the peptide D-JNKI-1 would ensure the presence of a greater number of projections in search of the target and therefore the possibility of obtaining a more efficient and more consistent reorganization, which hopefully can give an opportunity to a functional recovery.

\section{Conclusion}

Despite the evolutionary complexity of the central nervous system, our study has demonstrated that in response to early alterations of physiological development mechanisms an important organizational response comes in cortical and subcortical structures. Probably the aim of the morphological alterations observed is to anatomically compensate the losses caused by injury. However, we observed that physiological phenomena such as that of blindsight cause spontaneously an anatomical reorganization process which manages to balance, albeit weakly, the loss of functionally due to an irreversible damage.

The intent of these studies is to try to use to our advantage the possibilities that result from plastic potentials that the nervous system has, to a greater or lesser extent, during all of its life. By the use of inhibitors, such as D-JNKI-1 we have shown that as a result of a lesion the neuronal death can be reduced and that a greater number of cells can be kept alive, each of which is potentially capable of emitting projections able to regroup to compensate the losses caused by the injury. Thus increasing pharmacologically the plastic potential of the nervous system in response to adverse events it is plausible to think that it can also be obtained the maintenance of a greater functional efficiency, in case of such serious injuries to be considered so far cause of definitive impairment. We are probably still far from a possible pharmacological approach of this kind, but the first evidences emerged are auspicious to continue in the future researches to obtain very satisfactory results in both anatomical and functional fields.

Furthermore, the role of pharmacological JNK inhibition has been recently studied as an alternative therapy for prevention and treatment of age related macular degeneration [23].

A special aspect of combined study to increase, a harbinger of interesting and productive feedback, could be the comparison between experimental animal models and the findings in patients studied with functional magnetic resonance [24].

\section{Competing Interests}

All authors declare that we have no conflict of interest. 


\section{References}

1 Kluver H (1936) An analysis of the effects of the removal of the occipital lobes in monkeys. J Psychol 2: 49-61.

2 Keating EG (1975) Effects of prestriate and striate lesions on the monkey's ability to locate and discriminate visual forms. Exp Neurol 47: 16-25.

3 Mohler CW, Wurtz RH (1977) Role of striate cortex and superior colliculus in visual guidance of saccadic eye movements in monkeys. J Neurophysiol 40: 74-94.

4 Schilder P, Pasik T, Pasik P (1971) Extrageniculostriate vision in the monkey. II. Demonstration of brightness discrimination. Brain Res 32: 383-398.

5 Zihl J, Von Cramon D (1985) Visual field recovery from scotoma in patients with postgeniculate damage. A review of 55 cases. Brain 108: 335-365.

6 Chokron S, Perez C, Obadia M, Gaudry I, Laloum L, et al. (2008) From blindsight to sight: cognitive rehabilitation of visual field defects. Restor Neurol Neurosci 26: 305-320.

7 Christensen MS, Kristiansen L, Rowe JB, Nielsen JB (2008) Actionblindsight in healthy subjects after transcranial magnetic stimulation. Proc Natl Acad Sci USA 105: 1353-1357.

8 Vakalopoulos C (2005) A theory of blindsight--the anatomy of the unconscious: a proposal for the koniocellular projections and intralaminar thalamus. Med Hypotheses 65: 1183-1190.

9 Repici M, Atzori C, Migheli A, Vercelli A (2003) Molecular mechanisms of neuronal death in the dorsal lateral geniculate nucleus following visual cortical lesions. Neuroscience 117: 859-867.

10 Cowan WM, Fawcett JW, O'Leary DD, Stanfield BB (1984) Regressive events in neurogenesis. Science 225: 1258-1265.

11 Innocenti GM (1995) Exuberant development of connections, and its possible permissive role in cortical evolution. Trends Neurosci 18: 397-402.

12 O'Leary DD, Koester SE (1993) Development of projection neuron types, axon pathways, and patterned connections of the mammalian cortex. Neuron 10: 991-1006.
13 Innocenti GM (1991) The development of projections from cerebral cortex. In: Ottoson D, Autrum H, Perl ER, Schmidt RF, Shimazu H, Willis WD (eds) Progress in Sensory Physiology, vol.12. Springer, Berlin, Heidelberg.

14 Briggs F, Usrey WM (2008) Emerging views of corticothalamic function. Curr Opin Neurobiol 18: 403-407.

15 Vercelli A, Repici M, Garbossa D, Grimaldi A (2000) Recent techniques for tracing pathways in the central nervous system of developing and adult mammals. Brain Res Bull 51: 11-28.

16 Clascá F, Angelucci A, Sur M (1995) Layer-specific programs of development in neocortical projection neurons. Proc Natl Acad Sci USA 92: 11145-11149.

17 Arimatsu Y, Ishida M (2002) Distinct neuronal populations specified to form corticocortical and corticothalamic projections from layer $\mathrm{VI}$ of developing cerebral cortex. Neuroscience 114: 1033-1045.

18 Negyessy L, Gal V, Farkas T, Toldi J (2000) Cross-modal plasticity of the corticothalamic circuits in rats enucleated on the first postnatal day. Eur J Neurosci 12: 1654-1668.

19 Hattox AM, Nelson SB (2007) Layer $V$ neurons in mouse cortex projecting to different targets have distinct physiological properties. J Neurophysiol 98: 3330-3340.

20 Jones EG (2009) Synchrony in the interconnected circuitry of the thalamus and cerebral cortex. Ann N Y Acad Sci 1157: 10-23.

21 Vercelli AE, Cracco CM (1994) Effects of eye enucleation on NADPHdiaphorase positive neurons in the superficial layers of the rat superior colliculus. Brain Res Dev Brain Res 83: 85-98.

22 Deschênes M, Veinante P, Zhang ZW (1998) The organization of corticothalamic projections: reciprocity versus parity. Brain Res Brain Res Rev 28: 286-308.

23 Du H, Sun X, Guma M, Luo J, Ouyang H, et al. (2013) JNK inhibition reduces apoptosis and neovascularization in a murine model of agerelated macular degeneration. Proc Natl Acad Sci USA 110: 2377 2382.

24 Ajina S, Rees G, Kennard C, Bridge H (2015) Abnormal contrast responses in the extrastriate cortex of blindsight patients. J Neurosci 35: 8201-8213. 